\title{
Deber eine Classe von Integralen mit geschlossener Integrationscurve.
}

Von

L. PoohHammer in Kiel.

Hat' eine mehrdeutige Function $F(w)$ die Form.

$$
F(w)=\left(w-\alpha_{v}\right)^{\beta_{v}} f(w) \text {, }
$$

wo $f(w)$ in der Umgebung des Punktes $w=\alpha_{v}$ eindeutig ist, so hesitzt das Integral

$$
\int_{\alpha_{v}}^{x}(w-x)^{\lambda} F(w) d w,
$$

(1) $x$ gleichzeitig als Parameter und als obere Grenze vorkemnit, die Eigenschaft, den constanten Factor $e^{2 \pi i\left(\beta_{y}+\lambda\right)}$ aufzunehmen, wenn $x$ einen positiven Umlauf um den Punkt $\alpha_{\nu}$ ausführt*). Dieser Satz gestattet eine Erweiterung, indem gewissen Integralen mit geschlossener Integrationscurve eine ähnliche Eigenschaft auch im Fall der Umkreisung mehrerer singulärer Punkte zukommt. Man betrachtet im Folgenden ein Integral $\omega(x)$ der Function $(w-x)^{2} F(w)$, genommen nach $w$, dessen Integrationsweg $S$ in dem Punkte $x$ beginnt und endigt und eine beliebige Anzahl von singulären Punkten der Fủnetion $F(w)$ umschliesst. Die Curve $S$, die sich selbst nicht schneiden mö́ge, begrenze ein Flächenstück, auf welchem die singulären Punkte wan $\alpha_{2}, \ldots, \alpha_{m}$ der Function $F(w)$ liegen, und $F(w)$ habe die Form (1) $F(w)=\left(w-\alpha_{1}\right)^{\beta_{1}}\left(w-\alpha_{2}\right)^{\beta_{2}} \ldots\left(w-\alpha_{m}\right)^{\beta_{m}} H(w)$,

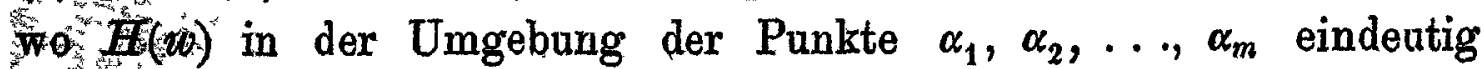
hlèbt. - Beschreibt dann die Variable $x$ einen positiven Umlauf um

\footnotetext{
1) Cfr. die Abh. des Herrn Hossenfelder "Ueber die Integration einer linearen Differentialgleichung $n^{\text {ter }}$ Ordnung" im $4^{\text {ten }}$ Bande dieser Annalen, sowie die Abh. des Verfassers "Ueber eine Classe von Functionen einer complexen Variablen, welche die Form bëstimmter Integrale haben" (\$ 2) im $104^{\text {ten }}$ Bande des Crelle'schen Journáls.
} 
das ganze von der Curve $S$ eingeschlossene Flächenstück, so dass die Punkte $\alpha_{1}, \alpha_{2}, \ldots, \alpha_{m}$, jedoch keine anderen singulären Punkte der Function $F(w)$, umkreist werden, so nimmt das Integral $\omega(x)$, wie in dem nachstehenden $\S 1$ gezeigt wird, den Factor

$$
e^{2 \pi i\left(\beta_{1}+\beta_{2}+\cdots+\beta_{m}+\lambda\right)}
$$

auf. Der Satz lässt sich auf mehrfache bestimmte Integrale von entsprechender Form ausdehnen. Der reelle Bestandtheil der Constante $\lambda+1$ wird in $\S 1$ als positiv vorausgesetzt, da das Integral $\omega(x)$ sonst divergent ist.

Genügt $\lambda$ der letzteren Bedingung nicht, so bildet man in der Art, wie der Verfasser in dem Aufsatze „Ueber ein Integral mit doppeltem Umlauf "*) angegeben hat, ein Integral $\Omega(x)$, dessen Integrationsweg aus zwei Umläufen (einem positiven und einem negativen) um den Punkt $x$ und zwei Umläufen um die Gesammtheit der Punkte $\alpha_{1}, \alpha_{2}, \ldots, \alpha_{m}$ besteht. Dieses Integral, welches in $\$ 2$ behandelt wird, nimmt ebenfalls, wenn $x$ einen positiven Umlauf um $\alpha_{1}, \alpha_{2}, \ldots, \alpha_{m}$ macht, den Factor $e^{2 \pi i\left(\beta_{1}+\beta_{2}+\cdots+\beta_{m}+\alpha\right)}$ auf. Ist der reelle Theil von $\lambda+1$ positiv, so unterscheiden sich $\omega(x)$ und $\Omega(x)$ nur durch einen constanten Factor. Das Integral $\Omega(x)$ bebält für jeden endlichen Werth von $x$, der nicht zu den singulären Werthen von $F(w)$ gehört, und für beliebige Werthe der in $F(w)$ vorkommenden Constanten einen bestimmten Sinn. Das in $\$ 2$ abgeleitete Resultat ist im Falle $m=1$ zugleich eine Ergänzung des anfänglich genannten, auf das Integral $\int_{\alpha_{v}}^{x}(w-x)^{\lambda} F(w) d w$ bezüglichen Satzes, da auch das letztere durch . ein Integral mit doppeltem Umlauf zu ersetzen ist, sobald dié reellen Theile der Constanten $\beta_{v}+1$ und $\lambda+1$ negativ werden.

*) Diese Annalen, Band 35, pag. 470.

Ich darf nicht unterlassen, zu erwahnen, dass Herr C. Jordan in dem bereits im Jahre 1887 veröffentlichten $3^{\text {ten }}$ Bande -seines "Cours d'Analyse de l'École Polytechnique" (Paris, Gauthier-Villars), der mir bisher unbekannt geblieben war, neben anderen Integralen mit gesohlossenem Integrationswege auch Integrale mit Doppelumlauf, anwendet $(\$ 193$ u. f.). Ferner wird in $\S 208$ des genannten Bandes gezeigt, dass das Integral der Function $t^{-1}(1-t)^{q-1}$, wenn man als Integrationsweg einen Doppelumblauf um die Punkte 0 und 1 wählt, gleich dem Producto aus $\left(1-e^{2 \pi i p}\right)\left(1-e^{2 \pi i q}\right)$ und dem Enler'schen Integralo erster Art ist. In Bezug auf einen Theil der in meinen Abhandlungen "Ueber ein Integral mit doppeltem Umlauf" und "Zur Theorie der Euler'schen Integrale" (Band 35 dieser Annalen, pag. 495) enthaltenen Sätze - die ich übrigens in druckfertiger Form schon im Frühjahr 1887 Fachgenossen vorgelegt habe kommt also Herrn C. Jordan dio Priorität $z$ a. 
$\S 1$.

Man verbinde die singulären Punkte $\alpha_{1}, \alpha_{2}, \ldots, \alpha_{m}$ der in (1) angegebenen Function $F(w)$ durch eine gebrochene, sich selbst nicht schneidende Linie $\mathfrak{A}$ (Fig. 1), so dass eine Umkreisung der Linie $\mathfrak{A}$ mit der Umkreisung der $m^{\prime}$ Punkte $\alpha_{1}, \alpha_{2}, \ldots, \alpha_{m}$ identisch ist. Dann kann das in der Einleitung definirte Integral $\omega(x)$ - nach $\S 1$ des érwähnten Aufsatzes „Ueber ein Integral mit doppeltem Umlauf" (Bd. 35 dieser Ann.) - kurz durch

$$
\omega(x)=\int_{x}^{(\mathfrak{( n )}}(w-x)^{\lambda} F(w) d w,
$$

oder, was dasselbe ist, durch

bezeichnet werden.

$$
\boldsymbol{\omega}(x)=\int_{x}^{\overline{(\alpha}^{\left(\alpha_{1}, \alpha_{2}, \cdots, \alpha_{m}\right)}}(w-x)^{\lambda} F(w) d w
$$

Da der reelle Theil der Constante $\lambda+1$ als positiv vorausgesetzt "wird, so tragen die dem Punkte $x$ unmittelbar benachbarten Theile des Integrationsweges nur unendlich wenig zum Werthe des Integrals $\omega(x)$ bei. Man darf also ein unendlich kleines Stück der Curve $S$ tahe dem Punkte $x$ fortlassen und auf diese Weise die geschlossene Integrationscurve in eine offene verwandeln. An der unteren Integralgrenze werde ein bestimmter Werth der Potenz $(w-x)^{\lambda}$ und ein bestimmter Werth der Function $F(w)$ fixirt. Derjenige Ausdruck, in "welchen der 'gewählte' Zweig der Function $F(w)$ übergeht, wenn w längs $S$ den Umlauf -um $\alpha_{1}, \alpha_{2}, \ldots, \alpha_{m}$ ausgeführt hat, soll durch $F_{1}(w)$ bezeichnet werden.

Es sei $x_{1}$ ein beliebiger Werth von $x$, ferner $x_{2}$ ein von $x_{1}$ unendlich wenig verschiedener Werth. Eine Curve $x_{1} s x_{2}$ (Fig. 1), die unter Umkreisung der Punkte $\alpha_{1}, \alpha_{2}, \ldots, \alpha_{m}$ vom Punkte $x_{1}$ in positiver Drehungsrichtung zum Punkte $x_{2}$ führt, wird wiederum kurz durch $S$ bezeichnet. Die Function $\omega\left(x_{1}\right)$ ist (abgesehen von dem unendlieh kleinen Betrag, welcher dem kurzen Bogenstück $x_{2} x_{1}$ entspricht) gleieh dem längs dieser Curve $S$ genommenen Integral

$$
\omega\left(x_{1}\right)=\int_{x_{1}}^{x_{2}}\left(w-x_{1}\right)^{n} F(w) d w .
$$

Es handelt sich darum, die stetigen Aenderungen von $\omega(x)$ in dem Ealle zu zumitteln, dass die Grösse $x$ rom Punkte $x_{1}$ aus einen positiven Umlauf um die Punkte $\alpha_{1}, \alpha_{2}, \ldots, \alpha_{m}$ macht. Da $x$ sowohl untere als obere Integralgrenze in $\omega(x)$ ist, so tritt der Weg; den $x$

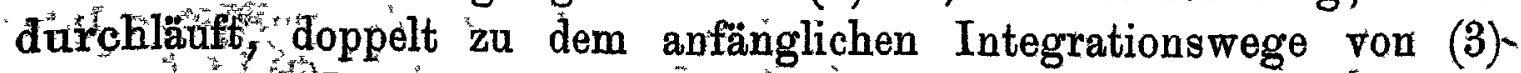
hinzu; ausserdem ändert sich, weñn $x$ variirt, die zu integrirende Function, da sie $x$ als Parameter enthält. 
Von $x_{1}$ aus werde (im positiven Sinne) eine ausserhalb $S$ bleibende Curve $x_{1} r_{1} \xi_{1}$ bis zu einem dicht bei $x_{1}$ liegenden Punkte $\xi_{1}$, und ebenso von $x_{2}$ aus eine der letzteren benachbarte Curve $x_{2} r_{2} \xi_{2}$ gezogen (Fig. 1). Die vier Punkte $x_{1}, x_{2}, \xi_{1}, \xi_{2}$ sollen einander unendlich nahe sein. Die Curren $x_{1} r_{1} \xi_{1}$ und $x_{2} r_{2} \xi_{2}$, die man schliesslich zusammenfallen lässt, geben den Weg der Variable $x$ an. Es wird vorausgesetzt, dass auf dem Flächenstück zwischen $x_{1} s x_{2}$ und $x_{2} r_{2} \xi_{2}$ keine singulären Punkte dẹ Function $F(w)$ liegen. Betrachtet man für irgend einen der Curve $x_{1} s x_{2}$ angehörigen Punkt $w$

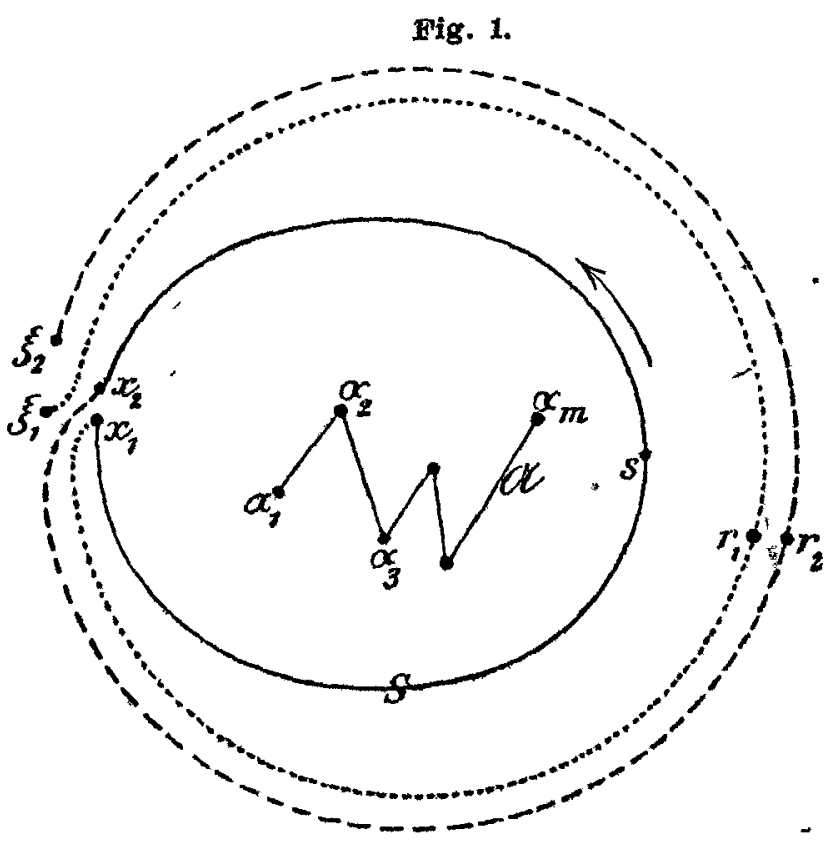
die Potenz $(w-x)^{\lambda}$, während $x$ die Curve $x_{1} r_{1} \xi_{1}$ durchläuft, so besteht, von einem unendlich kleinen Unterschied abgesehen, die Gleichung

$$
\left(w-\xi_{1}\right)^{\lambda}=e^{2 \pi i \lambda}\left(w-x_{1}\right)^{\lambda},
$$

da $x$ den Punkt $w$ im positiven Sinne umkreist hat.

Man bezeichnet durch $\omega\left(\xi_{1}\right)$ das Integral

$$
\omega\left(\xi_{1}\right)=\int_{\xi_{1}}^{\xi_{2}}\left(w-\xi_{1}\right)^{2} F(w) d w,
$$

dessen Integrationsweg die Linie $\xi_{1} r_{1} x_{1} s x_{2} r_{2} \xi_{2}$ ist. Die Grösse $\omega\left(\xi_{1}\right)$ stellt denjenigen Werth von $\omega(x)$ dar; "welcher aus $\omega(x)$ schtiesslich erhalten wird, wenn $x$ die Curve $x_{1} r_{1} \xi_{1}$ durchläuft. Der Integrationsweg von $\omega\left(\xi_{1}\right)$ zerfällt in die dreí Abschnitie $\xi_{1} r_{1} x_{r}, x_{1} s x_{2}$ und $x_{2} r_{2} \xi_{2}$. Für die Punkte $w$ des mittleren Abschnittes $x_{1} s, x_{2}$, wèleher den Integrationsweg von $\omega\left(x_{1}\right)$ angiebt, ist die Gleighung (4) anzawenden; die Werthe der Function $F(w)$ sind in diesen Punkten dienämlichen, wie bei dem Integral $\omega\left(x_{1}\right)$. Das in $\omega\left(\xi_{1}\right)$ enthíltène Intâgral längs der Curve $x_{1} s x_{2}$ bat also den Werth $e^{2 \pi i \lambda} \omega\left(x_{1}\right)$. Jedoch' hebt sich dieser Bestandtheil von $\omega\left(\xi_{1}\right)$, wie eine einfache Ueberlegung zeigt, gegen das Integral längs der Curve $\xi_{1} r_{1} x_{1}$ fort.:

$\mathrm{Da}$ in $\omega\left(\xi_{1}\right)$ der Punkt $x_{1}$ kein singulärer Punkt der zu integrirenden Function ist, so kann der Integrationsweg von $\omega\left(\xi_{1}\right)$ in der Art vereinfacht werden, dass statt der ztwêt ersten Abschnitte $\xi_{1} r_{1}$ und $x_{1} s x_{2}$ der geradlinige, unendlich "kleine Weg $\xi_{1} x_{2}$ gewählt wird. Letzterer liefert aber (da $\lambda+1>0$ ist) nur einen zu vernachlässigenden 
Beitrag zu dem Werthe von $\omega\left(\xi_{1}\right)$. Mithin ist $\omega\left(\xi_{1}\right)$ gleich dem längs $x_{2} r_{2} \xi_{2}$ genommenen Integral.

Im Punkte $w=x_{2}$ und in den folgenden Punkten $w$ der Curve $x_{2} r_{2} \xi_{2}$ bat die zu integrirende Function den Werth

$$
\left(w-\xi_{1}\right)^{\lambda} F_{1}(w), \quad=e^{2 \pi i \lambda}\left(w-x_{1}\right)^{\lambda} F_{1}(w) .
$$

'Denn der jetzt anzuwendende Zweig der Function $F(w)$ wird durch $F_{1}(w)$ bezeichnet, und die Gleichung (4) bleibt gültig, da die Werthe der Potenz $\left(w-\xi_{1}\right)^{2}$ für die Punkte $w$ der Curve $x_{2} r_{2} \xi_{2}$ sich stetig an die Werthe anschliessen, die zur Curve $x_{1} s x_{2}$ gehören. Demnach wird für $\omega\left(\xi_{1}\right)$ die Gleichung

\section{exhalten.}

$$
\omega\left(\xi_{1}\right)=e^{2 \pi i \lambda} \int_{x_{2}}^{\xi_{2}}\left(w-x_{1}\right)^{\lambda} F_{1}(w) d w
$$

Es soll nun die speciellere Voraussetzung gemacht werden, dass $F(w)$ eine Function sei, welche den constanten Factor $e^{2 \pi i \sigma}$ aufnimmt, wenn $w$ einen positiven Umlauf um die singulären Pünkte $\alpha_{1}, \alpha_{2}, \ldots, \alpha_{m}$ macht. Man setzt also

$$
F_{1}(w)=e^{2 \pi i \sigma} F(\dot{w})
$$

Dann ergiebt sich für $\boldsymbol{\omega}\left(\xi_{1}\right)$ der Ausdruck

$$
\omega\left(\xi_{1}\right)=e^{2 \pi i(\sigma+\lambda)} \int_{x_{2}}^{\xi_{2}}\left(w-x_{1}\right)^{2} F(w) d w .
$$

In dem rechts stehenden Integral kann der Integrationsweg $x_{2} r_{2} \xi_{2}$ durch den ursprünglichen Integrationsweg $S$ ersetzt werden, da auf dem Flächenstück $\mathrm{zwischen} \mathrm{den} \mathrm{beiden} \mathrm{Wegen} \mathrm{keine} \mathrm{singulären} \mathrm{Punkte}$ liegen. Auf diese Weise entsteht, wenn man die vier Punkte $x_{1}, x_{2}, \xi_{1}, \xi_{2}$ *chliesslich in einen Punkt zusammenfallen lässt, die Gleichung

$$
\omega\left(\xi_{1}\right)=e^{2 \pi i(\sigma+\lambda)} \omega\left(x_{1}\right) \text {. }
$$
die Form

Die Function $F(w)$ genügt der Bedingung (6) zunächst, wenn sie

$$
F(w)=\left(w-\alpha_{1}\right)^{\beta_{1}}\left(\bar{w}-\alpha_{2}\right)^{\beta_{2}} \ldots\left(w-\alpha_{m}\right)^{\beta_{m}} H(w)
$$

hat, woselbst $H(w)$ eine in der Umgebung der Punkte $\alpha_{1}, \alpha_{2}, \ldots, \alpha_{m}$ eindentige Function von $w$ bedeutet. In diesem Falle ist

und

$$
\begin{aligned}
& \omega(x)^{2}=\int_{x}^{\left(\alpha_{1}, \alpha_{2} \cdots, \alpha_{m}\right)}(w-x)^{2}\left(w-\alpha_{1}\right)^{\beta_{1}}\left(w-\alpha_{2}\right)^{\beta_{2}} \\
& \left(w-\alpha_{m}\right)^{\beta_{m}} H(w) d w_{\text {, }}
\end{aligned}
$$

wơdurch die Gleichung (7) in

$$
\sigma=\beta_{1}+\beta_{2}+\cdots+\beta_{m}
$$

$$
\omega\left(\xi_{1}\right)=e^{2 \pi i\left(\beta_{1}+\beta_{2}+\cdots+\beta_{m}+\lambda\right)} \omega\left(x_{i}\right)
$$

übergeht. Es, ist hiermit der in der Einleitung angegebene Satz bewiesen, dass das Integral (9) den constanten. Factor $e^{2 \pi i\left(\beta_{1}+\beta_{2}+\cdots+\beta_{m}+\lambda\right)}$ 
aufnimmt, wenn $x$ die Punkte $\alpha_{1}, \alpha_{2}, \ldots, \alpha_{m}$ im positiven Sinne umkreist. Die Constanten $\boldsymbol{\beta}_{1}, \boldsymbol{\beta}_{2}, \ldots, \boldsymbol{\beta}_{m}$ sind keinen Beschränkungen unterworfen, und die Constante $\lambda$ nur derjenigen, dass der reelle Theil ron $\lambda+1$ positiv sei.

Die Formeln (6) und (7) finden sodann auch auf den Fall Anwendung, wo die Function $F(w)$ selbst ein Integral von der Form (9) ist oder ein solches als Factor enthält. Es sei $\chi(w)$ ein zu (9) analoges Integral

$$
\begin{array}{r}
\chi(v)=\int_{w}^{\bar{\sigma}\left(\alpha_{1}, \alpha_{2}, \cdots, \alpha_{m}\right)}(v-w)^{\mu}\left(v-\alpha_{1}\right)^{\gamma_{1}}\left(v-\alpha_{2}\right)^{\gamma_{2}} \\
\ldots\left(v-\alpha_{m}\right)^{\gamma_{m}} h(v) d v
\end{array}
$$

und $F(w)$ die function

$$
F(w)=\left(w-\alpha_{1}\right)^{\beta_{1}}\left(w-\alpha_{2}\right)^{\beta_{2}} \ldots\left(w-\alpha_{m}\right)^{\beta_{m}} H(w) \chi(w),
$$

wo $H(w)$ und $h(v)$ in der Umgebung der Punkte $\alpha_{1}, \alpha_{2}, \ldots, \alpha_{m}$ als eindeutig vorausgesetzt werden. Dann nimmt in (6) die Constante $\sigma$ den Werth

$$
\beta_{1}+\beta_{2}+\cdots+\beta_{m}+\gamma_{1}+\gamma_{2}+\cdots+\gamma_{m}+\mu
$$

an. Folglich hat das Doppelintegral

$$
\omega(x)=\bar{\int}_{x}^{\left(\alpha_{12}, \alpha_{2}, \cdots, \alpha_{m}\right)}(w-x)^{\lambda} F(w) d w,
$$

in welchem $F(w)$ den Ausdruck (12) bedeutet, die Eigenschaft, den Factor

$$
e^{2 \pi i\left(\beta_{1}+\gamma_{1}+\beta_{2}+\gamma_{2}+\cdots+\beta_{m}+\gamma_{m}+\lambda+\mu\right)}
$$

aufzunehmen, wenn $x$ einen positiven Umlauf um die Punkte $\alpha_{1}, \alpha_{2}, \ldots, \alpha_{m}$ ausführt. In der nämlichen Art lässt sich der Satz auf vielfache Integrale von beliebiger Ordnung ausdehnen.

\section{\& 2.}

In den vorstehenden Rechnungen ist yorausgesetzt worden, dass der reelle Bestandtheil von $\lambda+1$ positiv sei. Genügt die-Constante $\lambda$ dieser Bedingung nicht, so wird, weil das Integral (2) divergirt, der in $\$ 1$ bewiesene Satz illusorisch. Derselbe kann indessen, wie gezeigt werden soll, als ein specieller Fall eines Satzes, der fur jeden Werth von $\lambda$ einen Sinn behält, aufgefasst werden. Statt des in (2) angegebenen Integrals $\omega(x)$ wird ein anderes Integral $\Omega(x)$ betrachtet, dessen Integrationsweg avis einem Doppelumfauf um $x$ und um $\alpha_{1}, \alpha_{2}, \ldots, \alpha_{m}$ besteht, und für das ebenfalls eine Gleichung von der Form (7) gilt.

Man verbinde wiederum die Punkte $\alpha_{1}, \alpha_{2}, \ldots, \alpha_{m}$, welche $m$ beliebige singuläre Argumente der Function $\mathcal{F}(w)$ darstellen, durch eirie gebrochene, sich nicht schneidende Linie $\mathfrak{A}$ und nenne $T$ ein Flächén- 
Stitiole; welches die ganze Linie $\mathfrak{A}$, jedoch keine anderen singulären Puniltè von $F(w)$ als $\alpha_{1}, \alpha_{2}, \ldots, \alpha_{m}$ enthält. Auf $T$ befinde sich auch der Punkt $x$; ferner sei $c$ ein beliebiger Punkt dieser Fläche, welcher keinem der Punkte $\alpha_{1}, \alpha_{2}, \ldots, \alpha_{m}, x$ unendlich nahe liegt und der Linie a nicht angehört. Von $c$ aus werde innerbalb $T$ eine geBoblossene Linie $c c_{1} c_{2} c^{\prime}$ gezogen, die man kurz $C$ nennt, und die den Punkt $x$ jedogh keinen der Punkte $\alpha_{1}, \alpha_{2}, \ldots, \alpha_{m}$ umschliesst. Ausser-

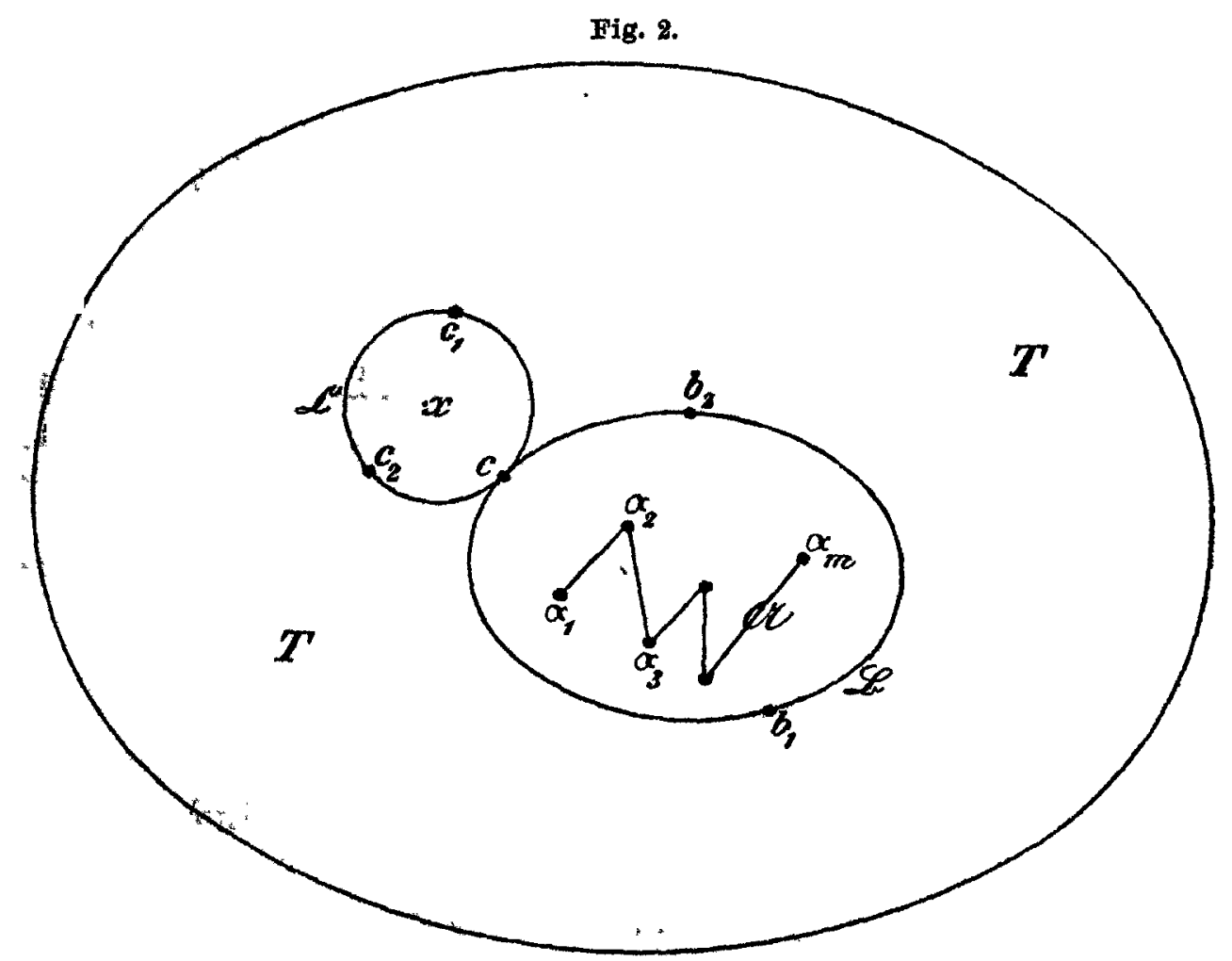

dem legt man eine geschlossene Curve $c b_{1} b_{2} c$, welche $\mathfrak{B}$ heissen möge, um- die Linie $\mathfrak{A}$; der Punkt $x$ befindet sich ausserbalb $\mathfrak{B}$. Es wird vorausgesetzt, dass die Punkte $c_{1}, c_{2}, b_{1}, b_{2}$ derartig liegen, dass der Weg $c c_{1} c_{2} c$ den positiven Umlauf um $x$, der Weg $c b_{1} b_{2} c$ den positiven Umlauf um $\alpha_{1}, \alpha_{2}, \ldots, \alpha_{m}$ bedeutet (Fig. 2).

7.

$$
(w-x)^{2} F(w) \text {, }
$$

Frassgan Integrationscurve sich aus den 4 auf einander folgenden Theilen

$$
c c_{1} c_{2} c, c b_{1} b_{2} c, c c_{2} c_{1} c, c b_{2} b_{1} c
$$

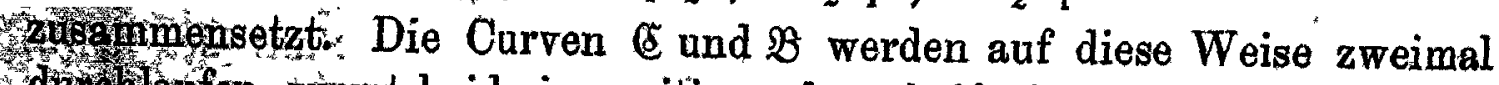
dur thaten, zurerst beide in positiver, dann beide in regativer Drehungs5. rielitug; Nach der obengenannten Abhandlung "Ueber ein Integral mit doppeltem Unlauf" wendet man für $\Omega(x)$ die abgekürzte Bezeichnung

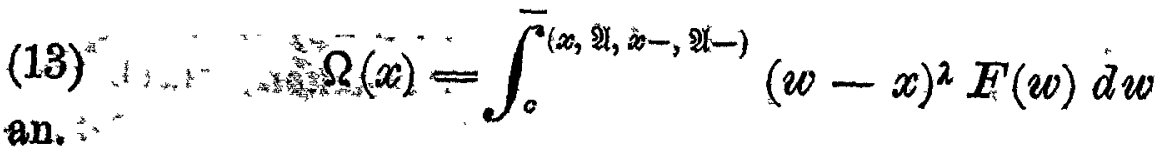


Es werde vorausgesetzt, dass die Function $F(w)$ die (in (6) angegebene) Eigenschaft habe, den Factor $e^{2 \pi i \sigma}$ aufzunehmen, wenn die Variable $w$ die Linie $\mathfrak{A}$ im positiven Sinne umkreist. Man nennt $\Phi(w)$ die zu integrirende Function in $\Omega(x)$, so dass

$$
\Phi(w)=(w-x)^{\lambda} F(w)
$$

ist. An der unteren Grenze $w=c$ des Integrals $\Omega(x)$ wird ein bestimmter Werth $\Phi(c)$ gewählt, der $\Phi_{0}$ heissen möge. Man bezeichnet ferner durch $N(x)$ das längs $c c_{1} c_{2} c$, sowie durch $M(x)$ das längs $c b_{1} b_{2} c$ erstreckte Integral von $\Phi(w)$, falls an der unteren Grenze $c$ dieser Integrale der Werth $\Phi_{0}$ als Anfangswerth von $\Phi(w)$ genommen wird. Analog zu (2) schreibt man abgekürzt

$$
\left\{\begin{array}{l}
N(x)=\bar{\int}_{0}^{(x)}(w-x)^{2} F(w) d w \\
M(x)=\int_{0}^{(\mathfrak{l})}(w-x)^{2} F(w) d w .
\end{array}\right.
$$

Das Integral $\Omega(x)$ lässt sich linear durch $N(x)$ und $M(x)$ ausdrücken. Bildet man die Gleichung

$$
\Omega(x)=\Omega_{1}(x)+\Omega_{2}(x)+\Omega_{3}(x)+\Omega_{4}(x),
$$

wo $\Omega_{1}(x), \Omega_{2}(x), \Omega_{3}(x), \Omega_{4}(x)$ die Integrale längs je eines der $4 \mathrm{Ab}$ schnitte des gegebenen Weges

bedeuten sollen, so ist

$$
c c_{1}^{\circ} c_{2} c, c b_{1} b_{2} c, c c_{2} c_{1} c, c b_{2} b_{1} c
$$

$$
\Omega_{1}(x)=N(x), \quad \Omega_{2}(x)=e^{2 \pi i \lambda} M(x) .
$$

Denn bei $N(x), M(x), \Omega_{1}(x)$ hat die Function $\Phi(w)$ an der unteren Integralgrenze den Werth $\Phi_{0}$, während man an der unteren Grenze von $\Omega_{2}(x)$, wegen der vorhergehenden Umkreisung des Punktes $x$, den Werth $e^{2 \pi i \lambda} \Phi_{0}$ für $\Phi(w)$ zu setzen hat. In $\Omega_{3}(x)$ gehört zur unteren Grenze der Werth $e^{2 \pi i(\lambda+\sigma)} \Phi_{0}$, zur oberen der Werth $e^{2 i \pi \sigma} \Phi_{0}$; am Endpunkte des Integrationsweges von $\Omega_{4}(x)$ nimmt die Function $\Phi(w)$ wieder den Werth $\Phi_{0}$ an. Indem man, unter Multiplication mit -1 , bei $\Omega_{3}(x)$ und $\Omega_{4}(x)$ die Integrationscurve umkehrt, ergiebt sich

so dass für $\Omega(x)$ die Gleichung

$$
\Omega_{3}(x)=-e^{2 \pi i \sigma} N(x), \quad \Omega_{4}(x)=-M(x),
$$
erhalten wird.

$$
\Omega(x)=\left(1-e^{2 \pi i \sigma}\right) N(x)+\left(e^{2 \pi i \lambda}-1\right) M(x)
$$

Um zu untersuchen, in welcher Weise die Grösse $\Omega(x)$ sich ändert, wenn die Variable $x$ einen positiven Umlauf um die Linie $\mathfrak{A}$ ausführt, reicht es wegen dér Gleichung (16) aus, das Verhalten der Grössen $M(x)$ und $N(x)$ im gedachten Falle festzustellen. Man darf 
voraússsetzen, dass die Curve, anf welcher $x$ bei Umkreisung der Linie $\mathfrak{A}$ fortschreitet, den Integrationsweg von $M(x)$ vollständig umschliesst, da letzterer auf eine der Linie $\mathfrak{A}$ beliebig nahe kommende Curve zusammengezogen werden kann. Dann wird, indem die Variable $x$ ihre Curve durchläuft, in jedem Element des Integrals $M(x)$ die Grösse $w$ von $x$ umkreist, worans folgt, dass der schliessliche Werth von $M(x)$ gleich dem Product aus dem anfänglichen Werthe und der Constante $e^{2 \pi i \lambda}$ ist. Bei $N(x)$ modificirt sich, wenn $x$ in der angegebenen Weise variirt, auch der Integrationsweg, da derselbe der $x$-Curve ausweichen muss*). Unm diese Aenderung zu verfolgen, soll zunächst die $x$-Curve näher bezeichnet werden.

Es sei, wie im vorigen Paragraphen, $x_{1}$ der anfängliche Werth von $x$, und $\xi$ ein unendlich nahe bei $x_{1}$ liegender Punkt; $x$ durchlaufe die Curve $x_{1} r \xi$ (die punktirte Linie in Fig. 3), welche den gegebenen Integrationsweg (c) des Integrals $N(x)$ in den Punkten $d_{2}$ und $d_{1}$ schneiden möge. Nan nennt $c_{1}$ und $c_{1}^{\prime}$ zwei Punkte von $\mathfrak{E}$, welche nicht weit von $d_{1}$,entfernt sind, und zwischen denen $d_{1}$ liegt; èbenso

Fig. 3.

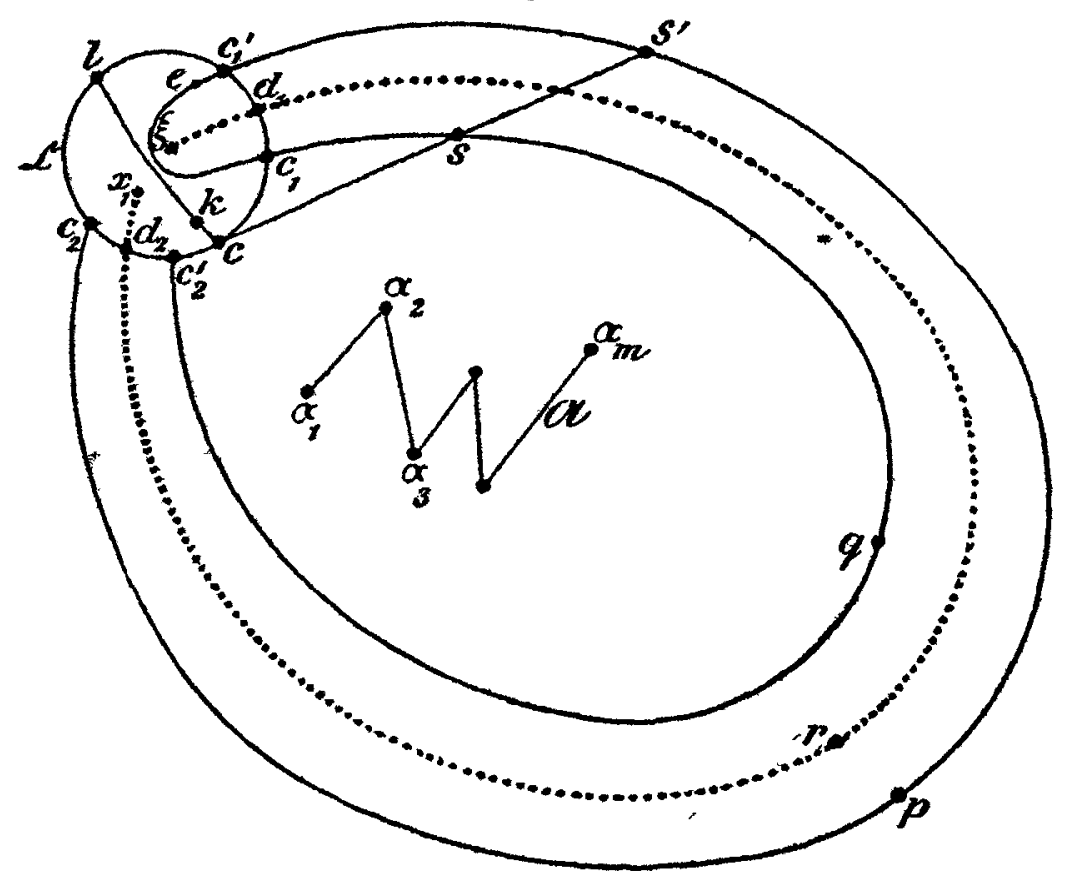

befinde sich $d_{2}$ zwischen den benachbarten Punkten $c_{2}$ und $c_{2}^{\prime}$ der Linie $c$. Es sèi ferner $l$ ein Pankt von $c$, welcher $c$ gegenüber liegt, in dem Sinne, dass er von $c$ durch $d_{1}$ und durch $d_{2}$ getrennt ist. In genaaerex Bezeichnung ist $c$ hiernach die Curve $c c_{1} d_{1} c_{1}{ }^{\prime} l c_{2} d_{2} c_{2}^{\prime} c$. Wenn $x$ atif der Curve $x_{1} r \xi$ bis zum ersten Schnittpunkte $d_{2}$ gelangt ist, so moss in Integral $N(x)$ die Variable $w$ den Punkt $d_{2}$ umgehen,

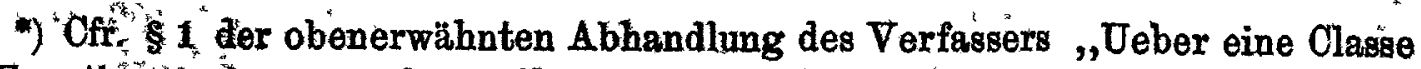
von Function nè einer complexen Variablen, welche die Form bestimmter Integraile haben", Crelle's Journal, Bd. 104. 
damit die $x$-Curve und der Integrationsweg sich nicht schneiden, und bei dem weiteren Fortschreiten von $x$ wiederholt sich (wegen der vorausgesetzten Stetigkeit der Aenderung) fortwährend dieses Zurückweichen des Integrationsweges vor der $x$-Curve. Statt des directen Weges von $c_{2}$ zu $c_{2}^{\prime}$ (auf der Curve (5) erhält man daher (Fig. 3) für die Variable $w$ einen Weg

$$
c_{2} p c_{1}^{\prime} e c_{1} q c_{2}^{\prime}
$$

welcher den Curvenbogen $d_{2} r d_{1} \xi$ von beiden Seiten umgiebt. In ähnlicher Weise weicht auch der Theil $c c_{1} d_{1} c_{1}^{\prime} l$ des Integrationsweges (5) dem Wege von $x$ aus. Man ziehe vom Punkte $c$ eine Linie $c k l$, welche zwischen $x_{1}$ und $\xi$ hindurchgeht; dieselbe tritt an Stelle des Weges $c c_{1} d_{1} c_{1}^{\prime} l$, da die $x$-Curve den Integrationsweg gewissermassen vor sich herschiebt. Also ist, nachdem $x$ die Curve $x d_{2} r d_{1} \xi$ durchlaufen hat, der ursprüngliche Integrationsweg $c c_{1} d_{1} c_{1}^{\prime} l_{2} d_{2} d_{2}^{\prime} c$ des betrachteten Integrals durch den Integrationsweg

$$
c k l c_{2} p c_{1}^{\prime} e c_{1} q c_{2}^{\prime} c
$$

zu ersetzen. Die anfänglichen Werthe der Integrale $M(x)$ und $N(x)$ sollen kurz durch $M\left(x_{1}\right)$ und $N\left(x_{1}\right)$, die schliesslichen Werthe derselben durch $M(\xi)$ und $N(\xi)$ bezeichnet werden. Bei $M\left(x_{1}\right)$ und $N\left(x_{1}\right)$ sei wiederum $\Phi_{0}$ der Werth der Function $\Phi(w)$ an der unteren Integralgrenze $w=c$.

Die Elemente des Integrals $N(x)$, welche der unteren Grenze. $w=c$ benachbart sind, nehmen den Factor $e^{2 \pi i \lambda}$ auf, wenn $x$ die Curve $x_{1} r \xi$ durchläuft. Denn letztere umschliesst die betreffenden Punkte $w$, so dass (abgesehen von einem unendlich kleinen Unterschiede) die - Potenz $(w-\xi)^{\lambda}$ gleich $e^{2 \pi i \lambda}\left(w-x_{1}\right)^{\lambda}$ zu setzen ist. Wegen der Stetigkeit von $\Phi(w)$ innerhalb des bestimmten Integrals überträgt sich dieser Factor $e^{2 \pi i \lambda}$ auf sämmtliche Elemente desselben.

Da im Integral $N(\xi)$ der Punkt $x_{1}$ kein singulärer ist, só kann man statt des Abschnitts $c k l c_{2} p$ des Integrationsweges einen kürzeren Weg von $c$ nach $p$ wählen. Andererseits sollen "gewisse Strecken zum Integrationswege hinzugefügt werden.' Es' sei $s$ ' ein beliebiger Punkt des Integrationsweges zwischen $p$ und $c_{1}^{\prime}$ (Fig. 3); man verbindet $c$ und $s^{\prime}$ durch eine Linie $c s s^{\prime}$, welche zwischen $\mathfrak{A}$ und $\xi$ hindurchgeht und den Bogen $c_{1} q$ des Integrationsweges in einem Punkte $s$ schneiden möge. Die Einschaltung der zwei Strecken $s^{\prime} s c$ und $c s s^{\prime}$ in den Integrationsweg ändert, da die Integrale längs $s^{\prime} s c$ und $c s s^{\prime}$ sich gegenseitig aufheben, den Werth von $N(\xi)$ nicht. Ebenso darf man zu dem letzten Theil des Integrationswegs noch einmal die Strecken sc und $c s$ (welche unmittelbar auf einander folgen sollen) hinzufügen. Mit Rücksicht hierauf kann der Integrationsweg , $\operatorname{ron} N(\xi)$ aus folgenden 3 Theilen zusammengesetzt werden

$$
c k l c_{2} p s^{\prime} s c, \quad c s s^{\prime} c_{1}^{\prime} e c_{1} s c_{2}, c s q c_{2}^{\prime} c \text {. }
$$


Den erste Theil stellt einen. Umlauf um die Linie $\mathfrak{A}$ dar; das Integral längs desselben ist (bis auf eine unendlich kleine Diffehenz) gleich dem Ausdruck

$$
e^{2 \pi i \lambda} M\left(x_{1}\right),
$$

da es sich von dem aben definirten Integral $M\left(x_{1}\right)$ nur dadurch unterscheidet, dass an der unteren Integralgrenze der Werth der zu integrirenden Function nicht $\Phi_{0}$, sondern $e^{2 \pi i \lambda} \Phi_{0}$ ist. Das Integral längs $e s s^{\prime} c_{1}^{\prime} e c_{1} s c$ stimint mit dem Producte

$$
e^{2 \pi i(\sigma+\lambda)} N\left(x_{1}\right)
$$

thereîn. Denn einerseits ist der singuläre Punkt $\xi$, der vom Integrationswege umkreist wird, von $x_{1}$ nur unendlich wenig verschieden, andererseits ist an der unteren Integralgrenze der Werth $e^{2 \pi i(\sigma+\lambda)} \Phi_{0}$ anzuwenden, weil $F(w)$ in Folge des Umlaufs um die Linie $\mathfrak{A}$ den Factor $e^{2 \pi i \sigma}$ anfgenommen hat. Am Anfangspunkte der Strecke $c s q c_{2}{ }^{\prime} c$ hat $\Phi(w)$ den Werth $e^{2 \pi i(\sigma+2 \lambda)} \Phi_{0}$, am Endpunkte derselben den Werth $e^{4 \pi i \lambda} \Phi_{0}$, wie sich aus den Drehungsrichtungen des Umlaufs um $\xi$ und des zweiten Umläufs um $\mathfrak{A}$ unmittelbar ergiebt. Indem man bei dem Integral längs $c s q c_{2}{ }^{\prime} c$ unter Aenderung des Vorzeichens den Integrationsweg umkehrt, findet man dasselbe gleich dem Producte

$$
-e^{4 \pi i \lambda} M\left(x_{1}\right) \text {. }
$$

Aưf diese Weise entstehen für $\boldsymbol{M}(\xi)$ und $N(\xi)$ die Gleichungen

$$
\left\{\begin{array}{l}
M(\xi)=e^{2 \pi i \lambda} M\left(x_{1}\right), \\
N(\xi)=e^{2 \pi i \lambda}\left(1-e^{2 \pi i \lambda}\right) M\left(x_{1}\right)+e^{2 \pi i(\sigma+\lambda)} N\left(x_{1}\right) .
\end{array}\right.
$$

Für $\Omega(\xi)$ bat man nach (16) den Ausdruck

$$
\Omega(\xi)=\left(1-e^{2 \pi i \dot{\sigma}}\right) N(\xi)+\left(e^{2 \pi i \lambda}-1\right) M(\xi) .
$$

Setzt man für $M(\xi)$ und $N(\xi)$ die in (17) erhaltenen Werthe ein, so entsteht die Gleichung

oder

$$
\begin{aligned}
& \Omega(\xi)=e^{2 \pi i(\sigma+\lambda)}\left(1-e^{2 \pi i \sigma}\right) N\left(x_{1}\right) \\
& \quad+e^{2 \pi i \lambda}\left[\left(1-e^{2 \pi i \sigma}\right)\left(1-e^{2 \pi i \lambda}\right)+e^{2 \pi i \lambda}-1\right] M\left(x_{1}\right)
\end{aligned}
$$

$$
\Omega(\xi)=e^{2 \pi i(\sigma+\lambda)}\left[\left(1-e^{2 \pi i \sigma}\right) N\left(x_{1}\right)+\left(e^{2 \pi i \lambda}-1\right) M\left(x_{1}\right)\right] .
$$

Da aber nach (16)

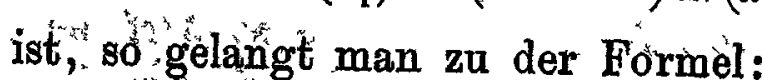

$$
\Omega\left(x_{1}\right)=\left(1-e^{2 \pi i \sigma}\right) N\left(x_{1}\right)+\left(e^{2 \pi i \lambda}-1\right) M\left(x_{1}\right)
$$

$$
\Omega(\xi)=e^{2 \pi i(\sigma+\lambda)} \Omega\left(x_{1}\right) .
$$

Die Function $\Omega(x)$ nimmt also, wie behauptet wurde, den Factor $e^{2 \pi i(\sigma+\lambda)}$ auf, wenn die Variable $x$ einen positiven Umlauf um die Punkte $\alpha_{1}, \alpha_{2}, \ldots, \alpha_{m}$ macht. 
Der Werth des Integrals $\Omega(x)$ ist von der Wahl des Punktes $c$, in welchem der Integrationsweg beginnt und endigt, unabhängig. Man beweist dies, indem man der Curve $(5)$ eine besondere Gestalt giebt. Wird der Punkt $c$ mit einem beliebigen anderen Punkte $c$ des Flächenstücks $T$ durch eine Linie $\Lambda$, die innerhalb $T$ bleibt und die Linie $\mathfrak{A}$ nicht schneidet, verbunden, und von $c$ eine geschlossene Curve $\mathfrak{D}$ um den Punkt $x$ gezogen (Fig. 4), so kann man die zuvor angewendete Curve 5 durch die Linie Fig. 4. $\Lambda$, die Linie $\mathfrak{D}$ und die in umgekehrter Richtung zum zweiten Male durchlaufene Linie $\Lambda$ ersetzen. Hierdurch wird das Integral $N(x)$ gleich dem Trinom

$$
\int_{0}^{c} \Phi(w) d w+\int_{c}^{(x)} \Phi(w) d w+\int_{c}^{0} \Phi(w) d w .
$$

Wie oben gezeigt wurde, hat $\Phi(w)$ am Endpunkte des ganzen Integrationsweges von $\Omega(x)$ denselben Werth, wie am Ausgangspunkte, so dass das erste der Theilintegrale, in welche man $\Omega(x)$ zerlegt, sich wieder stetig an das letzte anschliesst. In Folge dessen darf man bei der genannten Zerlegung das Integral

$$
\int_{c}^{c} \Phi(w) d w
$$

zum letzten Summandus machen und erhält hierdurch für $\Omega(x)$ den Ausdruck

$\Omega(x)=\bar{\int}_{c}^{(x)} \Phi(w) d w+\bar{\int}_{c}^{(\mathfrak{x})} \Phi(w) d w+\bar{\int}_{c}^{(x-)} \Phi(w) d w+\bar{\int}_{c}^{(\mathfrak{x}-)} \Phi(w) d w_{j}$ welcher sich von dem früheren Ausdruck der Grösse $\Phi(x)$ nur dadurch unterscheidet, dass der Punkt $c$ an dierstelle des Punktes $c$ getreten ist. Ist der reelle Bestandtheil der Constante $\lambda+1$ pósitiv, so kann man (Fig. 2) den Punkt $c$ unendlich nahe an den Punkt $x$ heranrücken lassen und die Dimensionen der Curve (5 verschwindend klein nehmen. Hierdurch wird $N(x)$ unendlich klein, und $M(x)$ mit dem in (2) bezeichneten Integral $\omega(x)$ identisch. Aus (16), ergiebt, sich dann die Gleichung

$$
\Omega(x)=\left(e^{2 \pi i \lambda}-1\right) \omega(x),
$$

welche die Beziehung "zwisctien den Integralểi $\Omega(x)$ und $\omega(x)$ in allen Fällen, wo $\omega(x)$ überhaupt einen bestimḿten Sinn hat, ausdrückt.

Kiel, im Mai 1890. 